\title{
OPEN Usefulness of computed tomography-measured psoas muscle thickness per height for predicting mortality in patients undergoing hemodialysis
}

\author{
Takahiro Yajima $^{1 凶}$, Maiko Arao ${ }^{1}$, Kumiko Yajima $^{2}$ \& Hiroshi Takahashi ${ }^{3}$
}

Computed tomography (CT)-measured psoas muscle thickness standardized for height (PMTH) has emerged as a promising predictor of mortality. The study aimed to investigate whether PMTH could accurately predict mortality in patients undergoing hemodialysis. We examined 207 patients (mean age: 63.1 years; men: $66.2 \%$ ) undergoing hemodialysis for more than 6 months in hospital affiliated clinic. PMTH was calculated at the $L 3$ vertebra level using CT. Patients were divided according to the PMTH cut-off points: $8.44 \mathrm{~mm} / \mathrm{m}$ in women and $8.85 \mathrm{~mm} / \mathrm{m}$ in men; thereafter, they were combined into low and high PMTH groups. PMTH was independently correlated with the simplified creatinine index $(\beta=0.213, P=0.021)$ and geriatric nutritional risk index $(\beta=0.295, P<0.0001)$ in multivariate regression analysis. During a median follow-up of 3.7 (1.8-6.4) years, 76 patients died, including 41 from cardiovascular causes. In the multivariate Cox regression analysis, low PMTH (adjusted hazard ratio, 2.48; 95\% confidence interval, 1.36-4.70) was independently associated with an increased risk of all-cause mortality. The addition of binary PMTH groups to the baseline risk model tended to improve net reclassification improvement $(0.460, p=0.060)$. In conclusion, PMTH may be an indicator of protein energy wasting and a useful tool for predicting mortality in patients undergoing hemodialysis.

Muscle wasting, i.e., the loss of muscle mass, is prevalent in patients with end-stage kidney disease undergoing hemodialysis ${ }^{1}$. Muscle wasting is a result of a negative protein balance caused by inflammation, increased protein catabolism, and insufficient energy and protein intake ${ }^{2}$. Thus, muscle wasting may be a common component of protein energy wasting ${ }^{3}$, which is defined as a loss of muscle and fat mass due to chronic inflammation, or sarcopenia ${ }^{4}$, which is characterized by low skeletal muscle mass with low muscle function. Both protein energy wasting and sarcopenia are prevalent and associated with increased mortality in patients undergoing hemodialysis ${ }^{3,5-7}$. Because muscle function, muscle strength, and physical performance are generally low in patients undergoing hemodialysis, the precise measurement of muscle mass is clinically important ${ }^{8}$. Surrogate methods such as bioelectrical impedance analysis and dual-energy X-ray absorptiometry are available in clinical practice; however, the accuracy of these methods can be affected by the patient's hydration status ${ }^{9,10}$. Thus, the European Consensus Statement recommends computed tomography (CT) as the gold standard method for the assessment of muscle mass, as it is not affected by hydration status ${ }^{4,11}$.

Several previous studies have reported that CT-measured indices such as the abdominal skeletal muscle index and psoas muscle mass index at the level of the third lumbar vertebra (L3) are widely used to diagnose sarcopenia or muscle wasting and predict mortality in patients with various cancers and chronic liver disease $\mathrm{e}^{12-14}$. However, specialized software, multiple attempts, and specific technical skills are required to measure these indices. Recently, CT-measured psoas muscle thickness per height (PMTH), defined as the largest transverse diameter of the right psoas muscle standardized for height, has been introduced as a simple measurable indicator of skeletal muscle mass for predicting mortality in patients with advanced liver disease ${ }^{15-19}$. However, in the field of nephrology, the use of CT for body composition analysis is limited owing to radiation exposure and high costs.

${ }^{1}$ Department of Nephrology, Matsunami General Hospital, Gifu 501-6062, Japan. ${ }^{2}$ Department of Internal Medicine, Matsunami General Hospital, Gifu 501-6062, Japan. ${ }^{3}$ Division of Medical Statistics, Fujita Health University School of Medicine, Aichi 470-1192, Japan. ${ }^{\circledR}$ email: yajima5639@gmail.com 


\begin{tabular}{|c|c|c|c|c|}
\hline & All patients $(N=207)$ & Low PMTH group $(N=95)$ & High PMTH group $(N=112)$ & $P$-value \\
\hline Age (years) & $63.1 \pm 13.6$ & $67.8 \pm 11.8$ & $59.1 \pm 13.8$ & $<0.0001$ \\
\hline Men (\%) & 66.2 & 55.8 & 75.0 & $<0.0001$ \\
\hline \multicolumn{4}{|l|}{ Underlying kidney disease } & 0.46 \\
\hline Diabetic kidney disease (\%) & 42.5 & 36.8 & 47.3 & \\
\hline Chronic glomerulonephritis (\%) & 30.9 & 33.7 & 28.6 & \\
\hline Nephrosclerosis (\%) & 19.8 & 20.0 & 19.6 & \\
\hline Others $(\%)$ & 6.8 & 9.5 & 4.5 & \\
\hline Hemodialysis vintage (years) & $2.1(0.9-5.1)$ & $2.1(0.8-5.6)$ & $2.0(1.0-5.0)$ & 0.89 \\
\hline Alcohol (\%) & 20.3 & 17.9 & 22.3 & 0.13 \\
\hline Smoking (\%) & 23.7 & 16.8 & 29.5 & 0.0028 \\
\hline Hypertension (\%) & 95.2 & 91.6 & 98.2 & 0.027 \\
\hline Diabetes (\%) & 45.4 & 41.1 & 49.1 & 0.23 \\
\hline History of CVD (\%) & 64.3 & 69.5 & 59.8 & 0.45 \\
\hline Dry weight $(\mathrm{kg})$ & $57.2 \pm 12.8$ & $51.9 \pm 10.6$ & $61.7 \pm 12.8$ & $<0.0001$ \\
\hline Height $(\mathrm{cm})$ & $160.5 \pm 8.7$ & $158.2 \pm 8.3$ & $162.5 \pm 8.7$ & $<0.0001$ \\
\hline Body mass index $\left(\mathrm{kg} / \mathrm{m}^{2}\right)$ & $22.1 \pm 4.0$ & $20.6 \pm 3.4$ & $23.3 \pm 4.2$ & $<0.0001$ \\
\hline Blood urea nitrogen $(\mathrm{mg} / \mathrm{dL})$ & $57.9 \pm 14.6$ & $55.6 \pm 16.3$ & $59.9 \pm 12.9$ & 0.023 \\
\hline Creatinine $(\mathrm{mg} / \mathrm{dL})$ & $9.5 \pm 3.1$ & $8.4 \pm 2.8$ & $10.4 \pm 3.0$ & $<0.0001$ \\
\hline Albumin $(\mathrm{g} / \mathrm{dL})$ & $3.7 \pm 0.5$ & $3.5 \pm 0.5$ & $3.8 \pm 0.3$ & $<0.0001$ \\
\hline Hemoglobin $(\mathrm{g} / \mathrm{dL})$ & $10.5 \pm 1.4$ & $10.4 \pm 1.5$ & $10.7 \pm 1.4$ & 0.16 \\
\hline Total cholesterol (mg/dL) & $150 \pm 33$ & $148 \pm 34$ & $152 \pm 33$ & 0.47 \\
\hline Uric acid (mg/dL) & $6.9 \pm 1.7$ & $6.8 \pm 1.9$ & $7.1 \pm 1.5$ & 0.19 \\
\hline Calcium (mg/dL) & $9.0 \pm 0.8$ & $8.9 \pm 0.9$ & $9.0 \pm 0.8$ & 0.27 \\
\hline Phosphorus (mg/dL) & $5.0 \pm 1.4$ & $4.7 \pm 1.4$ & $5.2 \pm 1.4$ & 0.020 \\
\hline iPTH (pg/mL) & $113(48-187)$ & $98(35-154)$ & $130(49-217)$ & 0.16 \\
\hline Glucose (mg/dL) & $144 \pm 60$ & $141 \pm 57$ & $147 \pm 63$ & 0.40 \\
\hline CRP (mg/dL) & $0.16(0.07-0.53)$ & $0.30(0.10-1.25)$ & $0.13(0.06-0.26)$ & 0.0004 \\
\hline Kt/V urea & $1.4 \pm 0.3$ & $1.4 \pm 0.3$ & $1.3 \pm 0.3$ & 0.0054 \\
\hline SCI (mg/kg/day) & $20.6 \pm 3.1$ & $19.3 \pm 2.7$ & $21.7 \pm 3.0$ & $<0.0001$ \\
\hline GNRI & $93.8 \pm 8.1$ & $90.1 \pm 8.8$ & $96.5 \pm 6.1$ & $<0.0001$ \\
\hline PMT (mm) & $15.5 \pm 6.4$ & $10.4 \pm 2.6$ & $19.9 \pm 5.3$ & $<0.0001$ \\
\hline PMTH $(\mathrm{mm} / \mathrm{m})$ & $9.6 \pm 3.8$ & $6.5 \pm 1.5$ & $12.2 \pm 3.1$ & $<0.0001$ \\
\hline
\end{tabular}

Table 1. Baseline characteristics of the study participants. $P M T H$ psoas muscle thickness per height, CVD cardiovascular disease, $i P T H$ intact parathyroid hormone, CRP C-reactive protein, SCI simplified creatinine index, GNRI geriatric nutritional risk index, $P M T$ psoas muscle thickness.

Only a few studies have examined the association between CT-measured sarcopenia indices and mortality in patients undergoing hemodialysis ${ }^{20-22}$. Although the use of PMTH for predicting mortality remains unclear, we hypothesized that low PMTH may be an increased risk of mortality in this population. It is clinically important to consider the pathophysiology which brings to lowering PMTH, therefore the relationships between PMTH and baseline variables were examined. Moreover, the present study aimed to investigate whether CT-measured PMTH can accurately predict all-cause and cardiovascular mortality in patients undergoing maintenance hemodialysis.

\section{Results}

Baseline characteristics. The baseline characteristics of the study participants are summarized in Table 1. The average age was $63.1 \pm 13.6$ years, and $66.2 \%$ were men. The median hemodialysis vintage was $2.1(0.9-5.1)$ years. History of cardiovascular disease was present in $64.3 \%$ of the participants. The geriatric nutritional risk index (GNRI), simplified creatinine index (SCI), and C-reactive protein (CRP) concentrations were 93.8 \pm 8.1 , $20.6 \pm 3.1 \mathrm{mg} / \mathrm{kg} / \mathrm{day}$, and $0.16(0.07-0.53) \mathrm{mg} / \mathrm{dL}$, respectively. The mean psoas muscle thickness (PMT) and PMTH were $15.5 \pm 6.4 \mathrm{~mm}$ and $9.6 \pm 3.8 \mathrm{~mm} / \mathrm{m}$, respectively. The PMT and PMTH were significantly higher in men than in women $(17.3 \pm 6.4 \mathrm{~mm}$ vs. $12.0 \pm 4.7 \mathrm{~mm}$ and $10.5 \pm 3.8 \mathrm{~mm} / \mathrm{m}$ vs. $7.8 \pm 3.0 \mathrm{~mm} / \mathrm{m}$, respectively; both $P<0.0001)$.

Agreements for PMT measurements. The intra- and inter-operator reproducibility of CT-measured PMT were assessed in a randomly selected sample of 30 patients; the measurements were performed in a blinded manner by two investigators (TY and MA). The intra-observer ICCs for the PMT measurements were 0.99 (95\% confidence interval [CI] 0.99-0.99) for TY and 0.99 (95\% CI 0.99-0.99) for MA. The interobserver intraclass correlation coefficient (ICC) for the PMT measurements was 0.98 (95\% CI 0.95-0.99). 


\begin{tabular}{|l|l|l|l|l|}
\hline \multirow{2}{*}{ Variables } & \multicolumn{2}{|l|}{ Univariate analysis } & \multicolumn{2}{l|}{ Multivariate analysis } \\
\cline { 2 - 5 } & r & $\boldsymbol{P}$-value & $\boldsymbol{\beta}$ & $\boldsymbol{P}$-value \\
\hline Age & -0.359 & $<0.0001$ & -0.093 & 0.26 \\
\hline Male sex & 0.335 & $<0.0001$ & 0.202 & 0.0025 \\
\hline Log CRP & -0.221 & 0.0014 & -0.010 & 0.87 \\
\hline SCI & 0.494 & $<0.0001$ & 0.213 & 0.021 \\
\hline GNRI & 0.473 & $<0.0001$ & 0.295 & $<0.0001$ \\
\hline
\end{tabular}

Table 2. Regression analyses of the associations between psoas muscle thickness per height and baseline variables. CRP C-reactive protein, SCI simplified creatinine index, GNRI geriatric nutritional risk index.

a

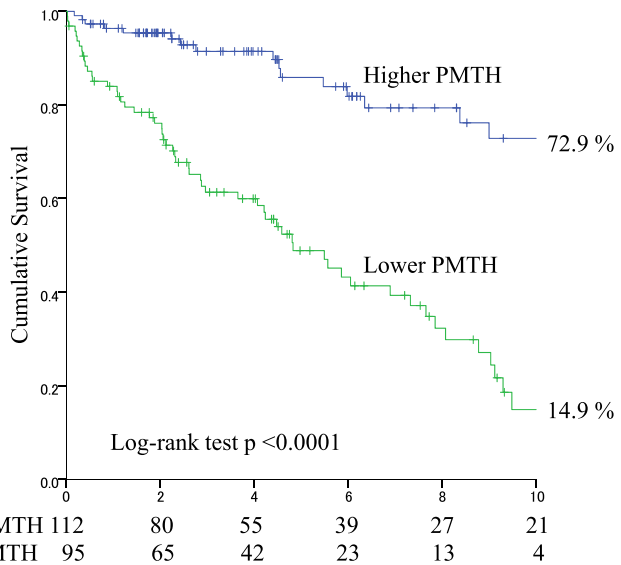

b

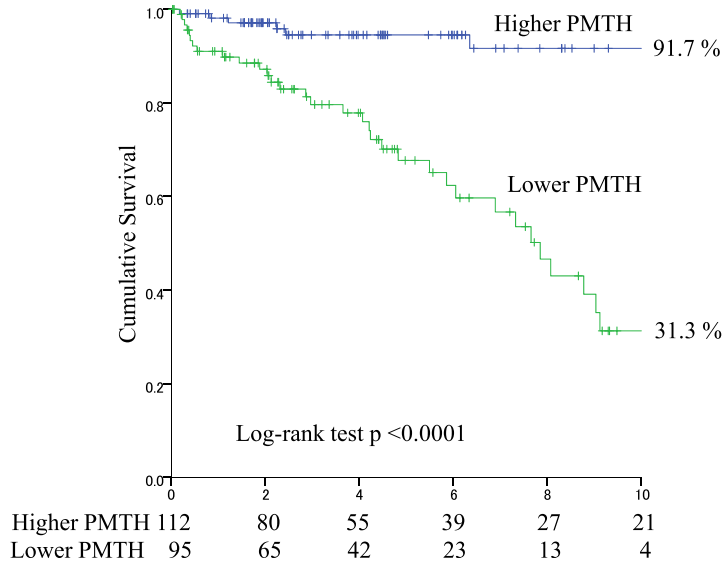

Figure 1. Kaplan-Meier survival curves. The all-cause mortality (a) and cardiovascular mortality (b) between the low PMTH and high PMTH groups were compared. PMTH psoas muscle thickness per height.

Associations between PMTH and baseline variables. In the univariate linear regression analysis, the PMTH was significantly and negatively associated with age and log CRP. Conversely, the PMTH was significantly and positively associated with sex (male), GNRI, and SCI. The correlation coefficients among all explanatory variables were less than 0.66 . The VIFs were less than 2.56. Multivariate regression analysis revealed that PMTH was independently associated with sex (male; $\beta=0.202, P=0.0025)$, SCI $(\beta=0.213, P=0.021)$, and GNRI $(\beta=0.295, P<0.0001)($ Table 2$)$.

Associations of PMTH with all-cause and cardiovascular mortality. During the median follow-up period of 3.7 (1.8-6.4) years, 76 patients died [cardiovascular disease (CVD), $\mathrm{n}=41$ (53.9\%); infection, $\mathrm{n}=19$ (25.0\%); cancer, $\mathrm{n}=4(5.3 \%)$; and others, $\mathrm{n}=12(15.8 \%)]$. In the univariate Cox proportional hazards analysis, the PMTH was a significant predictor of all-cause mortality [hazard ratio (HR), 0.78; 95\% CI, 0.71-0.84; $P<0.0001$ ]. Furthermore, the PMTH was a significant predictor of all-cause mortality in men (HR 0.72; 95\% CI 0.64-0.80, $P<0.0001$ ) and women (HR 0.82; 95\% CI 0.69-0.95; $P=0.0073$ ). To maximize the predictive value of PMTH for all-cause mortality in men and women, the receiver operating characteristic (ROC) analysis was performed and cut-off values of $8.44 \mathrm{~mm} / \mathrm{m}$ in women (sensitivity, 0.489 ; specificity, 0.751 ; area under the curve 0.634 ; $P=0.042$ ) and $8.85 \mathrm{~mm} / \mathrm{m}$ in men (sensitivity, 0.837 ; specificity, 0.765 ; area under the curve, $0.846 ; P<0.0001$ ) were obtained. The 10 -year survival rates were $72.9 \%$ for the high PMTH group and $14.9 \%$ for the low PMTH group $(P<0.0001)$ (Fig. 1). In multivariate Cox proportional hazards analysis adjusted for sex and age, history of CVD, SCI, GNRI, and log CRP, which were found to be significant factors in the univariate analysis, PMTH was an independent predictor of all-cause mortality (adjusted HR, 0.86; 95\% CI, 0.79-0.94; $P=0.0014$ ). There was no interaction between PMTH and sex for predicting mortality $(\mathrm{p}=0.26)$. A low PMTH (adjusted HR, 2.48; 95\% CI, 1.36-4.70; $P=0.0027$ ) was independently associated with an increased risk of all-cause mortality (Table 3 ). Similar results were obtained for cardiovascular mortality (Table 3 ).

Model discrimination. When compared with an established risk model including sex, age, a history of CVD, SCI, GNRI, and log CRP, the addition of binary PMTH did not improve Harrell's C-index for all-cause mortality $(0.828$ to $0.833, \mathrm{p}=0.76)$. However, the time dependent NRI and IDI tended to be improved $(0.460$, $\mathrm{p}=0.060$, and $0.064, \mathrm{p}=0.053$ ), respectively (Table 4 ). Similar results were obtained for cardiovascular mortality (Table 4). 


\begin{tabular}{|c|c|c|c|c|c|c|c|c|}
\hline \multirow[b]{2}{*}{ Variables } & \multicolumn{4}{|c|}{ Univariate analysis } & \multicolumn{4}{|c|}{ Multivariate analysis $^{\text {a }}$} \\
\hline & $\begin{array}{l}\text { Regression } \\
\text { coefficient }\end{array}$ & $\begin{array}{l}\text { Baseline } \\
\text { hazard }\end{array}$ & HR (95\% CI) & $P$-value & $\begin{array}{l}\text { Regression } \\
\text { coefficient }\end{array}$ & $\begin{array}{l}\text { Baseline } \\
\text { hazard }\end{array}$ & HR $(95 \% \mathrm{CI})$ & $P$-value \\
\hline \multicolumn{9}{|c|}{ All-cause mortality } \\
\hline $\begin{array}{l}\text { PMTH (con- } \\
\text { tinuous) }\end{array}$ & -0.25 & 7.81 & \begin{tabular}{|l}
0.78 \\
$(0.71-0.84)$
\end{tabular} & $<0.0001$ & -0.15 & 44.09 & \begin{tabular}{|l}
0.86 \\
$(0.79-0.94)$
\end{tabular} & 0.0014 \\
\hline Low PMTH & 1.64 & 0.34 & \begin{tabular}{|l|}
5.16 \\
$(3.01-8.82)$
\end{tabular} & $<0.0001$ & 0.91 & 15.08 & \begin{tabular}{|l|}
2.48 \\
$(1.36-4.70)$
\end{tabular} & 0.0027 \\
\hline \multicolumn{9}{|c|}{ Cardiovascular mortality } \\
\hline $\begin{array}{l}\text { PMTH (con- } \\
\text { tinuous) }\end{array}$ & -0.29 & 5.72 & \begin{tabular}{|l|}
0.75 \\
$(0.66-0.84)$
\end{tabular} & $<0.0001$ & -0.21 & 7.29 & \begin{tabular}{|l|}
0.81 \\
$(0.71-0.94)$
\end{tabular} & 0.0023 \\
\hline Low PMTH & 2.04 & 0.14 & \begin{tabular}{|l|}
$7.69(3.38-$ \\
$17.51)$
\end{tabular} & $<0.0001$ & 1.49 & 1.51 & \begin{tabular}{|l}
$4.23(1.77-$ \\
$11.47)$
\end{tabular} & $<0.0001$ \\
\hline
\end{tabular}

Table 3. Cox proportional hazards analysis of the psoas muscle thickness per height for prediction of allcause and cardiovascular mortality. HR hazard ratio, CI confidence interval, PMTH psoas muscle thickness per height. ${ }^{a}$ Adjusted for sex, age, history of cardiovascular disease, simplified creatinine index, geriatric nutritional risk index, and $\log \mathrm{C}$-reactive protein concentration, which were found to be significant in the univariate analysis.

\begin{tabular}{|c|c|c|c|c|c|c|}
\hline Variables & Harrell's C-index & $P$-value & Time dependent NRI & $P$-value & Time dependent IDI & $P$-value \\
\hline \multicolumn{7}{|l|}{ All-cause mortality } \\
\hline Established risk factors $^{\mathrm{a}}$ & 0.828 & & Reference & & Reference & \\
\hline + PMTH (continuous) & 0.834 & 0.79 & 0.396 & 0.080 & 0.056 & 0.053 \\
\hline + PMTH (low vs. high) & 0.833 & 0.76 & 0.460 & 0.060 & 0.064 & 0.053 \\
\hline \multicolumn{7}{|l|}{ Cardiovascular mortality } \\
\hline Established risk factors $^{\mathrm{a}}$ & 0.845 & & Reference & & Reference & \\
\hline + PMTH (continuous) & 0.855 & 0.83 & 0.454 & 0.073 & 0.075 & 0.073 \\
\hline + PMTH (low vs. high) & 0.854 & 0.49 & 0.663 & 0.073 & 0.121 & 0.060 \\
\hline
\end{tabular}

Table 4. Predictive accuracy of the psoas muscle thickness per height for all-cause and cardiovascular mortality using Harrell's C-index for Cox hazard model, time dependent NRI and time dependent IDI. NRI net reclassification improvement, IDI integrated discrimination improvement, $P M T H$ psoas muscle thickness per height. ${ }^{a}$ Sex, age, a history of cardiovascular disease, simplified creatinine index, geriatric nutritional risk index, and $\log$ C-reactive protein concentration.

\section{Discussion}

The main findings of the present study were that CT-measured PMTH was independently associated with SCI and GNRI, which were markers of protein energy wasting, and that a low PMTH was independently associated with an increased risk of all-cause and cardiovascular mortality in patients undergoing hemodialysis. Therefore, PMTH may be an indicator of protein energy wasting and, consequently, a simple and useful tool for accurately predicting mortality in this population.

In patients undergoing hemodialysis, muscle wasting may be partially explained by protein energy wasting ${ }^{2}$, a malnourished state of negative protein balance, characterized by the loss of body protein (muscle) and fuel reserves (fat) due to catabolic inflammation, which is prevalent and associated with an increased risk of mortality in this population ${ }^{3,23}$. We previously reported that high muscle mass and/or fat mass measured by bioelectrical impedance analysis are significantly associated with a decreased risk of all-cause mortality ${ }^{28}$. As a marker of protein energy wasting, GNRI, a simple objective nutritional assessment index that is calculated using only the serum albumin and actual/ideal body weight ratio, is widely used for patients undergoing hemodialysis ${ }^{24-28}$. The SCI, determined by age, sex, single pool Kt/V for urea, and pre-dialysis serum creatinine level, is used as a surrogate marker of muscle mass or as a valuable tool for observation of nutritional status in hemodialysis patients ${ }^{29-31}$. Tsai et al..$^{32}$ recently reported that SCI was an independent predictor of the presence of protein energy wasting. Moreover, Yamada et al. ${ }^{33}$ demonstrated that GNRI and SCI equally predicted all-cause mortality in hemodialysis patients. In the present study, CT-measured PMTH was independently and positively associated with both SCI and GNRI, suggesting that PMTH may be an indicator of protein energy wasting.

Recently, CT-measured PMTH has emerged as a marker of muscle wasting as well as a prognostic indicator of mortality in patients with liver disease ${ }^{15-19}$. Compared with conventional CT-based sarcopenic indices measured at the L3 level, this novel method has advantages such as no requirement of specific software or technical skills. However, it also has some issues that need to be addressed before it is standardized for use. Durand et al. ${ }^{15}$ and Huguet et al. ${ }^{16}$ reported that CT-measured PMTH at the umbilicus level predicts mortality in patients with liver cirrhosis. Considering sex-related differences in muscle distribution and structure, Gu et al. ${ }^{17}$ and Praktiknjo et al. ${ }^{18}$ proposed sex-specific cut-off points for PMTH at the umbilicus level. They demonstrated that the use of 
sex-specific cut-off points improves the prognostic value of PMTH in patients with cirrhosis. However, the level of the umbilicus is heterogeneously located from L3 to L5. Thus, Paternostro et al. ${ }^{19}$ recently proposed a sexspecific standardized measurement of PMTH at the L3 level, where conventional CT-based sarcopenic indices are measured, and showed that PMTH is superior to conventional CT-measured sarcopenic indices in predicting mortality of patients with cirrhosis.

CT may be the gold standard for the assessment of muscle mass ${ }^{4,11}$ even in patients undergoing hemodialysis because CT is not affected by the patient's hydration status. Several studies in Japan evaluated the association between CT-measured sarcopenic indices and prognosis in patients undergoing hemodialysis. Fukasawa et al. ${ }^{20}$ reported that the dry weight-adjusted thigh muscle area, but not the abdominal muscle area, is a predictor of mortality. Kurumisawa et al. ${ }^{21}$ demonstrated that the CT-measured psoas muscle index, assessed before cardiovascular surgery, is a predictor of long-term survival after cardiac surgery. Similarly, Takata et al..$^{22}$ reported that the CT-measured psoas muscle index is correlated with the bioelectrical impedance analysis-measured skeletal muscle mass index, where a low psoas muscle index is associated with an increased risk of mortality. Moreover, they suggested that the psoas muscle index assessed using CT can be an alternative to bioelectrical impedance analysis in patients undergoing hemodialysis. However, the relationship between CT-measured PMTH, a new CT-measured sarcopenic index, and mortality remains unknown. To our knowledge, our study is the first to analyze the relationship between CT-measured PMTH and mortality in patients undergoing hemodialysis. In the present study, a lower PMTH was independently associated with an increased risk of all-cause and cardiovascular mortality, even after adjusting for SCI and GNRI. In addition, the addition of PMTH on predicting model with established risk factors tended to improve the NRI and IDI, however, did not reach at statistical significance, probably due to small number of patients. These should be re-evaluated in larger population.

Our study has several limitations. First, the present retrospective, single-center study included a relatively small number of patients undergoing hemodialysis. Second, all enrolled patients were Japanese, who reportedly have a better prognosis that hemodialysis patients in the United States of America and Europe ${ }^{34}$. Thus, the results might not be generalizable to patients from other countries undergoing hemodialysis. Third, only PMTH data measured at enrolment were used for the analysis; therefore, any changes in these values during the longterm follow-up period were not considered. Further prospective, large-scale, multicenter studies are needed to validate our results.

In conclusion, CT-measured PMTH at the L3 level was independently associated with SCI and GNRI, and a low PMTH was independently associated with an increased risk of all-cause and cardiovascular mortality in patients undergoing hemodialysis. Therefore, PMTH may be an indicator of protein energy wasting and could be considered a simple and useful tool for accurately predicting mortality in this population.

\section{Methods}

Study participants. A total of 231 patients undergoing hemodialysis for longer than 6 months and who had undergone abdominal CT to detect early-stage renal cell carcinoma between January 2008 and December 2018 at the outpatient clinic of Matsunami General Hospital were screened. Twenty-four patients with a history of cancer were excluded; thus, 207 patients were ultimately enrolled in the present study. In our hospital, abdominal CT was performed as a screening test to detect early-stage renal cell carcinoma within 1 year after either the initiation of hemodialysis or transfer to our hospital. For this retrospective study, the requirement for informed consent was waived by the ethics committee of Matsunami General Hospital because patient data were anonymized. This study adhered to the principles of the Declaration of Helsinki, and the study protocol was approved by the ethics committee of Matsunami General Hospital (No. 484).

Data collection. The following data were collected from the medical charts of the study participants: age; sex; underlying kidney disease; hemodialysis vintage; history of alcohol use, smoking, hypertension, diabetes, and CVD; dry weight; and height. In this study, CVD included angina pectoris, myocardial infarction, heart failure, stroke, and peripheral artery disease. Hypertension was defined as the use of antihypertensive drugs or a systolic blood pressure $\geq 140 \mathrm{~mm} \mathrm{Hg}$ and/or a diastolic blood pressure $\geq 90 \mathrm{~mm} \mathrm{Hg}$ prior to hemodialysis. Diabetes was defined as the use of glucose-lowering medication or a history of diabetes. Blood samples were obtained prior to the initiation of a hemodialysis session with the patient in the supine position, and the laboratory data that were extracted for data analysis were from the month when the CT was performed.

The GNRI was calculated from serum albumin level, dry weight, and height as follows: GNRI $=14.89 \times$ serum albumin $(\mathrm{g} / \mathrm{dL})+41.7 \times(\text { dry weight/ideal body weight })^{25,35}$. Ideal body weight $(\mathrm{kg})$ was calculated as $22 \times$ [height $(\mathrm{m})]^{2}$. When dry weight was equal to or exceeded the ideal body weight, the ratio of dry weight to ideal body weight (dry weight/ideal body weight) was set to 1 . The SCI was calculated from sex, age, single pool Kt/V for urea, and serum creatinine level as follows: $\mathrm{SCI}(\mathrm{mg} / \mathrm{kg} / \mathrm{day})=16.21+1.12 \times[0$ for woman; 1 for man $]-0.06 \times$ age (years) $-0.08 \times$ single pool Kt/V for urea $+0.009 \times$ serum creatinine $(\mu \mathrm{mol} / \mathrm{L})^{29}$.

Measurement of PMTH. The PMT was determined using abdominal CT that was performed after a hemodialysis session using a wide-bore, 16-slice multidetector CT scanner (LightSpeed RT16; GE Healthcare, Waukesha, WI, USA), yielding 5-mm-thick slices. A cross-sectional CT image at the level of L3 was selected, and the transverse thickness of the right psoas muscle was measured using XTREK View (J-Mac System Inc., Sapporo, Japan). The transverse PMT was defined as the diameter of the psoas muscle perpendicular to the axial diameter, as previously reported ${ }^{15-19}$. The PMT was standardized for height to obtain the PMTH: PMTH $(\mathrm{mm} / \mathrm{m})=\operatorname{PMT}(\mathrm{mm}) /$ height $(\mathrm{m})$. 
Follow-up study. The primary endpoint was all-cause mortality, and the secondary endpoint was cardiovascular mortality. Patients were followed up until December 2019. In this study, follow-up period was defined as the interval from the date when CT was performed to the date of death or the date when transferred to another hemodialysis unit. Patients who were alive were censored in December 2019.

Statistical analysis. Normally distributed variables were expressed as means \pm standard deviations, while non-normally distributed variables were expressed as medians and interquartile ranges. The Shapiro-Wilk test and visual inspection were used to examine whether a variable is normally distributed. ROC analysis was performed to assess the sensitivity and specificity of PMTH for predicting 10-year all-cause mortality in each sex. The optimal sex-specific cut-off values of PMTH were determined at the point to maximize Youden's J statistic. Thereafter, patients were combined into low and high PMTH groups. The differences between the low and high PMTH groups were compared using Student's $t$-test or the Wilcoxon rank sum test for continuous variables and the chi-squared test for categorical variables. Intra- and interobserver reproducibility for the measurement of PMT using CT were evaluated using the ICC and 95\% CIs.

Univariate linear regression analysis was performed to examine the baseline factors associated with PMTH. Subsequently, multivariate regression analysis was performed using the factors found to be significant $(P<0.05)$ in the univariate analysis. As assumptions for multivariate linear regression analysis, continuous variables which were non-normally distributed were log-transformed. To confirm the multi-collinearity, correlation coefficients were calculated among all explanatory variables. Variance Inflation Factors (VIFs) were also calculated. The Kaplan-Meier method was used to estimate survival, and the difference of the survival was compared using the log-rank test. HRs and 95\% CIs for all-cause and cardiovascular mortality were examined using Cox proportional hazard regression analysis. Since the distribution of the PMTH values differed according to sex, interaction between PMTH and sex was examined, and sex was included in the multivariate analysis.

To determine whether the prediction of all-cause and cardiovascular mortality improved after the addition of PMTH to the baseline model with sex and the covariates that were found to be significant in the univariate analysis, Harrell's C-index for Cox regression model ${ }^{36,37}$, time dependent net reclassification improvement $(\mathrm{NRI})^{38}$ and time dependent integrated discrimination improvement (IDI) ${ }^{39}$ were calculated with likelihoodratio tests using $\mathrm{R}$ version 4.04. The NRI is a relative indicator of the number of patients for whom the predicted probability of mortality improves, whereas the IDI represents the average improvement in predicted probabilities for mortality after the addition of variables to the baseline model. All statistical analyses were performed using SPSS version 21 (IBM Corp., Armonk, NY, USA) and R version 4.04. Statistical significance was set at $P<0.05$.

Ethics declarations. This study adhered to the principles of the Declaration of Helsinki, and the study protocol was approved by the ethics committee of Matsunami General Hospital (No. 484). The requirement for informed consent was waived because patient data were anonymized.

\section{Data availability}

The dataset analyzed in the present study is available from corresponding author on reasonable request.

Received: 3 July 2021; Accepted: 13 September 2021

Published online: 24 September 2021

\section{References}

1. Giglio, J. et al. Association of sarcopenia with nutritional parameters, quality of life, hospitalization, and mortality rates of elderly patients on hemodialysis. J. Ren. Nutr. 28, 197-207 (2018).

2. Sabatino, A., Cuppari, L., Stenvinkel, P., Lindholm, B. \& Avesani, C. M. Sarcopenia in chronic kidney disease: What have we learned so far?. J. Nephrol. https://doi.org/10.1007/s40620-020-00840-y (2020).

3. Fouque, D. et al. A proposed nomenclature and diagnostic criteria for protein-energy wasting in acute and chronic kidney disease. Kidney. Int. 73, 391-398 (2008).

4. Cruz-Jentoft, A. J. et al. Sarcopenia: European consensus on definition and diagnosis: Report of the European Working Group on Sarcopenia in Older People. Age Ageing. 39, 412-423 (2010).

5. Isoyama, N. et al. Comparative associations of muscle mass and muscle strength with mortality in dialysis patients. Clin. J. Am. Soc. Nephrol. 9, 1720-1728 (2014).

6. Ren, H., Gong, D., Jia, F., Xu, B. \& Liu, Z. Sarcopenia in patients undergoing maintenance hemodialysis: Incidence rate, risk factors and its effect on survival risk. Ren. Fail. 38, 364-371 (2016).

7. Kim, J. K. et al. Impact of sarcopenia on long-term mortality and cardiovascular events in patients undergoing hemodialysis. Korean J. Intern. Med. 34, 599-607 (2019).

8. Bataille, S. et al. The diagnosis of sarcopenia is mainly driven by muscle mass in hemodialysis patients. Clin. Nutr. 36, 1654-1660 (2017).

9. Tangvoraphonkchai, K. \& Davenport, A. Changes in body composition following haemodialysis as assessed by bioimpedance spectroscopy. Eur. J. Clin. Nutr. 71, 169-172 (2017).

10. Pietrobelli, A., Formica, C., Wang, Z. \& Heymsfield, S. B. Dual-energy X-ray absorptiometry body composition model: Review of physical concepts. Am. J. Physiol. 271, E941-951 (1996).

11. Cruz-Jentoft, A. J. et al. Sarcopenia: Revised European consensus on definition and diagnosis. Age Ageing. 48, 601 (2019).

12. Shachar, S. S., Williams, G. R., Muss, H. B. \& Nishijima, T. F. Prognostic value of sarcopenia in adults with solid tumours: A metaanalysis and systematic review. Eur. J. Cancer. 57, 58-67 (2016).

13. Kim, G., Kang, S. H., Kim, M. Y. \& Baik, S. K. Prognostic value of sarcopenia in patients with liver cirrhosis: A systematic review and meta-analysis. PLoS ONE 12, e0186990 (2017).

14. Amini, B., Boyle, S. P., Boutin, R. D. \& Lenchik, L. Approaches to assessment of muscle mass and myosteatosis on computed tomography: A systematic review. J. Gerontol. A. Biol. Sci. Med. Sci. 74, 1671-1678 (2019).

15. Durand, F. et al. Prognostic value of muscle atrophy in cirrhosis using psoas muscle thickness on computed tomography. J. Hepatol. 60, 1151-1157 (2014). 
16. Huguet, A. et al. The psoas muscle transversal diameter predicts mortality in patients with cirrhosis on a waiting list for liver transplantation: A retrospective cohort study. Nutrition 51-52, 73-79 (2018).

17. Gu, D. H. et al. Clinical usefulness of psoas muscle thickness for the diagnosis of sarcopenia in patients with liver cirrhosis. Clin. Mol. Hepatol. 24, 319-330 (2018).

18. Praktiknjo, M. et al. Sarcopenia is associated with development of acute-on-chronic liver failure in decompensated liver cirrhosis receiving transjugular intrahepatic portosystemic shunt. Clin. Transl. Gastroenterol. 10, e00025 (2019).

19. Paternostro, R. et al. The value of different CT-based methods for diagnosing low muscle mass and predicting mortality in patients with cirrhosis. Liver. Int. 39, 2374-2385 (2019).

20. Fukasawa, H. et al. Lower thigh muscle mass is associated with all-cause and cardiovascular mortality in elderly hemodialysis patients. Eur. J. Clin. Nutr. 71, 64-69 (2017).

21. Kurumisawa, S. \& Kawahito, K. The psoas muscle index as a predictor of long-term survival after cardiac surgery for hemodialysisdependent patients. J. Artif. Organs. 22, 214-221 (2019).

22. Takata, T. et al. Feasibility of computed tomography-based assessment of skeletal muscle mass in hemodialysis patients. J. Nephrol. 34, 465-471 (2021).

23. Yajima, T., Yajima, K., Takahashi, H. \& Yasuda, K. The impact of abdominal fat levels on all-cause mortality risk in patients undergoing hemodialysis. Nutrients 10, 480 (2018).

24. Yajima, T., Arao, M., Yajima, K., Takahashi, H. \& Yasuda, K. The associations of fat tissue and muscle mass indices with all-cause mortality in patients undergoing hemodialysis. PLoS ONE 14, e0211988 (2019).

25. Takahashi, H. et al. Comparison of nutritional risk scores for predicting mortality in Japanese chronic hemodialysis patients. J. Ren. Nutr. 27, 201-206 (2017).

26. Yajima, T., Yajima, K. \& Takahashi, H. Impact of annual change in geriatric nutritional risk index on mortality in patients undergoing hemodialysis. Nutrients 12, 3333 (2020).

27. Yajima, T., Yajima, K., Takahashi, H. \& Yasuda, K. Combined predictive value of extracellular fluid/intracellular fluid ratio and the geriatric nutritional risk index for mortality in patients undergoing hemodialysis. Nutrients 11, 2659 (2019).

28. Yajima, T., Yajima, K. \& Takahashi, H. Association of the erythropoiesis-stimulating agent resistance index and the geriatric nutritional risk index with cardiovascular mortality in maintenance hemodialysis patients. PLoS ONE 16, e0245625 (2021).

29. Canaud, B. et al. Creatinine index as a surrogate of lean body mass derived from urea Kt/V, pre-dialysis serum levels and anthropometric characteristics of haemodialysis patients. PLoS ONE 9, e93286 (2014).

30. Hwang, W. et al. Comparison of creatinine index and geriatric nutritional risk index for nutritional evaluation of patients with hemodialysis. Hemodial. Int. 22, 507-514 (2018).

31. Canaud, B. et al. Clinical and predictive value of simplified creatinine index used as muscle mass surrogate in end-stage kidney disease haemodialysis patients-results from the international MONitoring Dialysis Outcome initiative. Nephrol. Dial. Transplant. 35, 2161-2171 (2020).

32. Tsai, M. T. et al. Comparison of simplified creatinine index and systemic inflammatory markers for nutritional evaluation of hemodialysis patients. Nutrients 30, 1870 (2021).

33. Yamada, S. et al. Geriatric nutritional risk index (GNRI) and creatinine index equally predict the risk of mortality in hemodialysis patients: J-DOPPS. Sci. Rep. 10, 5756 (2020).

34. Robinson, B. M., Bieber, B., Pisoni, R. L. \& Port, F. K. Dialysis Outcomes and Practice Patterns Study (DOPPS): Its strengths, limitations, and role in informing practices and policies. Clin. J. Am. Soc. Nephrol. 7, 1897-1905 (2012).

35. Yamada, K. et al. Simplified nutritional screening tools for patients on maintenance hemodialysis. Am. J. Clin. Nutr. 87, 106-113 (2008).

36. Harrell Jr, F.E. Quantifying predictive ability. in Regression Modeling Strategies with Applications to Linear Models, Logistic Regression, and Survival Analysis, 1st edn. 492-493. (Springer, 2001).

37. Schröder, M. S., Culhane, A. C., Quackenbush, J. \& Haibe-Kains, B. Survcomp: An R/Bioconductor package for performance assessment and comparison of survival models. Bioinformatics 27, 3206-3208 (2011).

38. Pencina, M. J., D’Agostino, R. B. \& Steyerberg, E. W. Extensions of net reclassification improvement calculations to measure usefulness of new biomarkers. Stat. Med. 30, 11-21 (2011).

39. Uno, H., Tian, L., Cai, T., Kohane, I. S. \& Wei, L. J. A unified inference procedure for a class of measures to assess improvement in risk prediction systems with survival data. Stat. Med. 32, 2430-2442 (2012).

\section{Acknowledgements}

All authors deeply thanks Takuma Ishihara, MSc, Innovative and Clinical Research Promotion Center, Gifu University Hospital (Gifu, Japan) for his important suggestion and supporting on statistical analysis.

\section{Author contributions}

T.Y., M.A., K.Y., and H.T. formulated the study concept and design. T.Y. and M.A. collected data. T.Y., M.A., K.Y., and H.T. analyzed and interpreted data. T.Y. and M.A. drafted the manuscript. T.Y., M.A., K.Y., and H.T. critically reviewed the content of the manuscript and revised it for important intellectual content. T.Y. and H.T. conducted statistical analysis. All authors read and approved the final manuscript.

\section{Funding}

The authors did not receive support from any organization for the submitted work.

\section{Competing interests}

The authors declare no competing interests.

\section{Additional information}

Correspondence and requests for materials should be addressed to T.Y.

Reprints and permissions information is available at www.nature.com/reprints.

Publisher's note Springer Nature remains neutral with regard to jurisdictional claims in published maps and institutional affiliations. 
(c) (i) Open Access This article is licensed under a Creative Commons Attribution 4.0 International cc) License, which permits use, sharing, adaptation, distribution and reproduction in any medium or format, as long as you give appropriate credit to the original author(s) and the source, provide a link to the Creative Commons licence, and indicate if changes were made. The images or other third party material in this article are included in the article's Creative Commons licence, unless indicated otherwise in a credit line to the material. If material is not included in the article's Creative Commons licence and your intended use is not permitted by statutory regulation or exceeds the permitted use, you will need to obtain permission directly from the copyright holder. To view a copy of this licence, visit http://creativecommons.org/licenses/by/4.0/.

(C) The Author(s) 2021 